九州大学学術情報リポジトリ

Kyushu University Institutional Repository

\title{
FINITE TREE AUTOMATA ON INFINITE TREES
}

Hayashi, Takeshi

College of General Education, Kyushu University

Miyano, Satoru

Department of Mathematics, Kyushu University

https://doi.org/10.5109/13369

出版情報: Bulletin of informatics and cybernetics. 21 (3/4), pp.71-82, 1985-03. Research Association of Statistical Sciences

バージョン :

権利関係 : 


\title{
FINITE TREE AUTOMATA ON INFINITE TREES
}

\author{
By
}

\author{
Takeshi HayAShI* and Satoru Mryano**
}

\begin{abstract}
Finite tree automata on infinite trees which move from the root infinitely are investigated through six types of acceptance conditions. Each condition is an extension of the one used for finite automata on $\omega$ words. We relate the six classes of infinite tree languages with the corresponding classes of $\omega$-languages defined by finite automata, and the relationship among these six classes is established.
\end{abstract}

\section{Introduction}

Rabin [6-8] introduced finite tree automata on infinite trees to study the problems of definability in monadic second-order theories. As by-products, he obtained several decidability results. He considered the acceptance conditions which are extensions of the ones studied in $[1,3]$. For $\omega$-languages, a series of acceptances by finite automata have been introduced and investigated $[1-4,8,10-12]$. These acceptance definitions for $\omega$-languages can be also naturally extended for the infinite tree acceptance by finite tree automata and we can define the classes of infinite tree languages defined by these finite tree automata. In this paper we deal with six types of acceptance conditions for finite tree automata on infinite trees which move from the root infinitely. Each condition uses a family of final sets for acceptance as was done in [3] for finite automata on $\omega$ words. The purpose of this paper is to establish the relationship among these infinite tree language classes. First it is proved that a single final set is sufficient for four types of acceptance definitions. We also prove an embedding theorem which relates the infinite tree language classes with the $\omega$-language classes. With the aid of this embedding theorem and a result due to Rabin [7], we classify the infinite tree language classes. A summary of the results is figured out in Fig. 1 in Section 5.

It is known that the nondeterministic infinite tree language class is larger than the deterministic one for any type of the acceptances [9]. To refine this result, we consider the nondeterministic degree which is defined to be the maximum number of possible transitions of a finite tree automaton. Then the determinism is regarded as the nondeterministic degree one. We show that the nonderministic degree gives a hierarchy between the nondeterministic class and the deterministic one for each acceptance definition. This is a property special to (infinite) tree languages. In addition to this observation, the results in this paper show that the class of infinite tree languages accepted by

* College of General Education, Kyushu University 01, Ropponmatsu, Fukuoka, 810 Japan.

** Department of Mathematics, Kyushu University 33, Fukuoka, 812 Japan. 
nondeterministic finite tree automata which accept in the manner of Rabin [6] is the most general one and this class may be considered as a natural extension of the class of $\omega$-regular languages [3], where deterministic finite automata are used as basis.

\section{Preliminaries}

Let $\Sigma_{k}=\{0,1, \cdots, k-1\}$ be an alphabet consisting of $k$ symbols. The infinite $k$-ary tree is the set $T_{k}=\Sigma_{k}^{*}$. The empty string $\varepsilon$ is the roof of $T_{k}$. For $x \in T_{k}, x 0, x 1, \cdots$, $x(k-1)$ are the sons of $x$ arranged from left to right. Note that $T_{k} \subset T_{k+1}$ for $k \geqq 1$. Let $\Sigma$ be a finite alphabet. An infinite k-ary tree over $\Sigma$ (ak-ary $\Sigma$-tree) is a function $t: T_{k} \rightarrow \Sigma$. The set of all $k$-ary $\sum$-trees is denoted by $\Sigma^{T_{k}}$. For $x, y$ in $T_{k}$, we say that $x$ is a prefix of $y$ denoted by $x \leqq y$ if $y=x z$ for some $z$ in $\Sigma_{k}^{*}$. The prefix relation gives a partial ordering on $T_{k}$. A path in $T_{k}$ is a subset $\pi \cong T_{k}$ satisfying (i) $\varepsilon \in \pi$, (ii) for $x \in \pi$, exactly one of $k$ sons $x 0, x 1, \cdots, x(k-1)$ belongs to $\pi$, (iii) $\pi$ is the smallest subset of $T_{k}$ satisfying (i) and (ii).

For a set $S,|S|$ denotes the cardinality of $S$ and $P(S)$ is the set of all subsets of S. For a function $f: X \rightarrow Y$ and a subset $X^{\prime}$ of $X, f \mid X^{\prime}$ denotes the restriction of $f$ to $X^{\prime}$.

Definition. A (nondeterministic) finite k-ary tree automation is a quintuple $M=$ $\left\langle S, \Sigma, \Delta, s_{0}, \mathcal{F}\right\rangle$, where

(1) $S$ is a finite set of states,

(2) $\Delta$ is a transition function $\Delta: S \times \Sigma \rightarrow P\left(S^{k}\right)-\{\phi\}$, where $S^{k}$ is the $k$-fold product of $S$,

(3) $S_{0}$ is a state in $S$ called the initial state,

(4) $F$ is a family of subsets of $S . F$ in $\mathscr{F}$ is called a final set and states in $F$ are called final states.

$M$ is said to be deterministic if $|\Delta(s, \sigma)|=1$ for each $s$ in $S$ and each $\sigma$ in $\Sigma$.

Let $M=\left\langle S, \Sigma, \Delta, s_{0}, \mathcal{F}\right\rangle$ be a finite $k$-ary tree automaton and let $t: T_{k} \rightarrow \Sigma$ be a $k$-ary $\Sigma$-tree. A run of $M$ on $t$ is a function $r: T_{k} \rightarrow S$ such that $r(\varepsilon)=s_{0}$ and for each $x$ in $T_{k},(r(x 0), r(x 1), \cdots, r(x(k-1))) \in \Delta(r(x), t(x))$. Let $\pi$ be a path in $T_{k}$. For a run $r: T_{k} \rightarrow S$ we define

$$
\operatorname{In}(r \mid \pi)=\{s \in S \mid s=r(x) \text { for infinitely many } x \text { in } \pi\} .
$$

Definition. A finite $k$-ary tree automaton $M=\left\langle S, \Sigma, \Delta, s_{0}, \mathscr{F}\right\rangle$ is said to accept a $k$-ary $\Sigma$-tree $t$ in the sense of $C_{i}(i=1, \cdots, 6)$ if there exists a run $r$ of $M$ on $t$ such that for each path $\pi \cong T_{k}$ there is a final set $F$ in $\mathscr{I}$ which satisfies the condition $C_{i}$ described below. A run $r$ satisfying the condition $C_{i}$ is called a $C_{i}$-accepting run of $M$ on $t$.

$\left(C_{1}\right) \quad \operatorname{In}(r \mid \pi) \cap F \neq \varnothing$.

$\left(C_{2}\right) \quad \operatorname{In}(r \mid \pi) \subseteq F$.

$\left(C_{3}\right) \quad r(\pi) \cap F \neq \varnothing$.

$\left(C_{4}\right) \quad r(\pi) \leqq F$.

$\left(C_{5}\right) \quad \operatorname{In}(r \mid \pi)=F$.

$\left(C_{6}\right) \quad r(\pi)=F$.

The acceptance defined by $C_{1}$ condition is sometimes called the acceptance in the 
sense of Büchi and the $C_{\tilde{0}}$-acceptance is called the acceptance in the sense of Muller.

Definition. For $i=1, \cdots, 6$, the set $L_{i}(M)$ of $k$-ary $\Sigma$-trees is defined as

$$
L_{i}(M)=\left\{t \in \Sigma^{T_{k}} \mid t \text { is accepted by } M \text { in the sencse of } C_{i}\right\} .
$$

We say that a set $L \subseteq \Sigma^{T_{k}}$ is accepted by $M$ in the sense of $C_{i}$ if $L=L_{i}(M)$.

Definition. For $i=1, \cdots, 6$, we define

(1) $\mathscr{R}_{i}^{(k)}=\left\{L_{i}(M) \mid M\right.$ is a nondeterministic finite $k$-ary tree automaton $\}$,

(2) $\mathscr{D}_{i}^{(k)}=\left\{L_{i}(M) \mid M\right.$ is a deterministic finite $k$-ary tree automaton $\}$.

In this paper we are concerned only with the classes for $k=1,2$. We denote $\Omega_{i}^{(1)}$ (resp. $\mathscr{D}_{i}^{(1)}$ ) by $\mathscr{R}_{i}$ (resp. $\mathscr{D}_{i}$ ). The classes $\mathscr{R}_{i}^{(2)}$ and $\mathscr{D}_{i}^{(2)}$ are specially denoted by $\mathscr{N}_{i}$ and $\mathscr{D} \mathscr{I}_{i}$, respectively. A binary $\Sigma$-tree is simply called a $\Sigma$-tree and a finite binary tree automaton is called a finite tree automaton. For $k=1$, this is just the case of finite automata on $\omega$-words $[1-4,8,10-12]$. In fact, $T_{1}=\{0\}^{*}$ and $\Sigma^{T_{1}}$ is the same as the set $\Sigma^{\omega}$ of $\omega$-words. A subset of $\Sigma^{T_{1}}$ is called an $\omega$-language. We call a finite unary tree automaton by a finite automaton. The reader is referred to $[2,11]$ for the terminologies on $\omega$-words and $\omega$-languages.

Finite automata on $\omega$-words have been extensively studied and the classes $\Re_{i}$ and $\mathscr{D}_{i}(i=1, \cdots, 6)$ of $\omega$-languages have been completely determined $[2,10-12]$. Table 1 summarizes the results on $\bigcap_{i}$ and $\mathscr{D}_{i}(i=1, \cdots 6)$. Here $R$ denotes the class of $\omega$-regular languages [3]. With respect to the product topology on $\Sigma^{\omega}$ based on the discrete topology on $\Sigma$, the classes in Table 1 are characterized as $G^{R}=G \cap R, F^{R}=F \cap R, G_{\delta}^{R}=$ $G_{\delta} \cap R$ and $F_{\sigma}^{R}=F_{\sigma} \cap R$, where $G($ resp. $F$ ) denotes the class of open (resp. closed) sets and $G_{\delta}\left(\right.$ resp. $\left.F_{\delta}\right)$ denotes the class of countable intersections (resp. unions) of open (resp. closed) sets. The inclusion relations among these classes are $G^{R} \varsubsetneqq G_{\delta}^{R} \varsubsetneqq R, F^{R} \varsubsetneqq F_{\sigma}^{R} \varsubsetneqq R$ and $G^{R} \cup F^{R} \varsubsetneqq G_{\tilde{o}}^{R} \cap R_{\sigma}^{R}$. We refer the reader to [4, 10-12] for more details on these classes.

Table 1

\begin{tabular}{ccccccc}
\hline & $C_{1}$ & $C_{2}$ & $C_{3}$ & $C_{4}$ & $C_{5}$ & $C_{6}$ \\
\hline $\mathscr{D}_{i}$ & $G_{\delta}^{R}$ & $F_{\sigma}^{R}$ & $G^{R}$ & $F^{R}$ & $R$ & $G_{\delta}^{R} \cap F_{\sigma}^{R}$ \\
$\Re_{i}$ & $R$ & $F_{\sigma}^{R}$ & $G^{R}$ & $F^{R}$ & $R$ & $F_{\sigma}^{R}$ \\
\hline
\end{tabular}

\section{Finite Tree Automata with a Single Final Set}

In this section we consider finite tree automata which have just one final set. In the literatures $[4,10]$, it has been known that a single final set is sufficient for the $\omega$-word acceptances by $C_{i}$ conditions for $i=1, \cdots 4$. The purpose of this section is to show that an analogue also holds for infinite tree acceptances.

A finite tree automaton $M=\left\langle S, \Sigma, \Delta, s_{0},\{F\}\right\rangle$ with a final set $F$ is also denoted by $M=\left\langle S, \Sigma, \Delta, s_{0}, F\right\rangle$.

THEOREM 3.1. Let $M=\left\langle S, \Sigma, \Delta, s_{0}, \mathscr{F}\right\rangle$ be a finite tree automaton. Then for each $i=1, \cdots, 4$, there exists a finite tree automaton $\tilde{M}=\left\langle\tilde{S}, \Sigma, \tilde{A}, \tilde{s}_{0}, \tilde{F}\right\rangle$ with a final set $\tilde{F}$ such that $L_{i}(M)=L_{i}(\tilde{M})$. Furthermore, if $M$ is deterministic, so is $\tilde{M}$.

Proof. The idea of the proof is due to [4, Theorem $4.1 ; 10$, Lemma 7]. 
CASE 1. $i=1,3: \tilde{M}$ is simply defined by $\tilde{M}=\left\langle S, \Sigma, \Delta, s_{0}, \tilde{F}\right\rangle$, where $\tilde{F}=\{s \mid s \in F$ for some $F$ in $\subseteq$.

CASE 2. $i=2: \tilde{M}$ is defined as follows :

(1) $\tilde{S}=\{(s, \tilde{\mathscr{F}}) \mid \tilde{\mathscr{I}} \subseteq \mathscr{F}$ and for each $F$ in $\tilde{\mathscr{I}}, s$ is in $F\}$.

(2) $\tilde{s}_{0}=\left(s_{0}, \varnothing\right)$.

(3) If $\left(s_{1}, s_{2}\right)$ is in $\Delta(s, \sigma)$, then for all $\varnothing \neq \tilde{I} \subseteq \mathscr{I}, \tilde{\mathcal{J}}((s, \tilde{\mathscr{I}}), \sigma)$ contains $\left(\left(s_{1},\{F \mid F\right.\right.$ $\left.\left.\left.\in \tilde{\mathscr{F}}, s_{1} \in F\right\}\right),\left(s_{2},\left\{F \mid F \in \tilde{\mathscr{F}}, s_{2} \in F\right\}\right)\right)$ and $\tilde{J}((s, \varnothing), \sigma)$ contains $\left(\left(s_{1},\left\{F \mid F \in \mathscr{F}, s_{1} \in F\right\}\right)\right.$, $\left.\left(s_{2},\left\{F \mid F \in \mathscr{F}, s_{2} \in F\right\}\right)\right)$.

(4) $\tilde{F}=\{(s, \tilde{\mathscr{I}}) \mid(s, \tilde{\mathscr{I}}) \in \widetilde{S}$ with $\tilde{\mathscr{F}} \neq \varnothing\}$.

Let $t: T_{2} \rightarrow \Sigma$ be a $\Sigma$-tree. First we establish a one-to-one correspondence between the runs of $M$ on $t$ and the runs of $\tilde{M}$ on $t$. Let $\tilde{r}: T_{2} \rightarrow \tilde{S}$ be an arbitrary run of $\tilde{M}$ on $t$. Then we associate it with a run $r: T_{2} \rightarrow S$ such that $r(x)=s$, where $\tilde{r}(x)=(s, \tilde{\mathscr{F}})$ for $x$ in $T_{2}$. By (3), we can see that $r$ is a run of $M$ on $t$. Conversely for a run $r: T_{2} \rightarrow S$ of $M$ on $t$, we associate it with a run $\tilde{r}: T_{2} \rightarrow \widetilde{S}$ of $\tilde{M}$ on $t$ which is defined as follows: $\tilde{r}$ is inductively defined. First we define $\tilde{r}(\varepsilon)=\left(s_{0}, \varnothing\right)$. Suppose that $\tilde{r}(x)=(s, \tilde{F})$ is defined. Then note that $r(x)=s$. Assume that in the run $r$ a transition $\left(s_{1}, s_{2}\right) \in$ $\Delta(s, t(x))$ is taken at the node $x$. Then if $\tilde{\mathcal{F}}=\varnothing$, the transition $\left(\left(s_{1},\left\{F \mid F \in \mathscr{F}, s_{1} \in F\right\}\right)\right.$, $\left.\left(s_{2},\left\{F \mid F \in \mathscr{F}, s_{2} \in F\right\}\right)\right)$ is taken at $x$ in the run $\tilde{r}$. Namely we define $\tilde{r}(x 0)=\left(s_{1},\{F \mid F\right.$ $\left.\left.\in \mathscr{F}, s_{1} \in F\right\}\right)$ and $\tilde{r}(x 1)=\left(s_{2},\left\{F \mid F \in \mathscr{F}, s_{2} \in F\right\}\right)$. If $\tilde{\mathscr{F}} \neq \varnothing$, then we put $\tilde{r}(x 0)=\left(s_{1},\{F \mid F\right.$ $\left.\left.\in \tilde{\mathscr{F}}, s_{1} \in F\right\}\right)$ and $\tilde{r}(x 1)=\left(s_{2},\left\{F \mid F \in \tilde{\mathscr{F}}, s_{2} \in F\right\}\right)$. Again by (3) we see that $\tilde{r}$ is a run of $\tilde{M}$ on $t$. By this correspondence we assume that $r$ and $\tilde{r}$ denote the runs corresponding to each other. To prove $L_{2}(M)=L_{2}(\tilde{M})$, it suffices to show that the following two statements are equivalent for each path $\pi \subseteq T_{2}$.

(5) There exists $F$ in $\mathscr{I}$ such that $\operatorname{In}(r \mid \pi) \leqq F$.

(6) $\operatorname{In}(\tilde{r} \mid \pi) \subseteq \tilde{F}$.

For a path $\pi=\left\{x_{0}<x_{1}<\cdots x_{n}<\cdots\right\}$, let $\tilde{r}\left(x_{n}\right)=\left(s_{n}, F_{n}\right)$ for $n \geqq 0$. Then there exists an integer $n_{0}$ such that $\operatorname{In}(r \mid \pi)=\left\{s_{n} \mid n \geqq n_{0}\right\}$ and $\operatorname{In}(\tilde{r} \mid \pi)=\left\{\left(s_{n}, \mathscr{F}_{n}\right) \mid n \geqq n_{0}\right\}$. Suppose that $\operatorname{In}(r \mid \pi) \subseteq F$ for some $F$ in $\mathscr{F}$. Then by the choice of $n_{0}$ we see that $s_{n}$ is in $F$ for all $n \geqq n_{0}$. If $\mathscr{F}_{n_{1}}=\varnothing$ for some $n_{1} \geqq n_{0}$, then by the choice of $n_{0}$ we see that $\mathscr{I}_{n}=\varnothing$ for infinitely many $n \geqq n_{1}$. As we observed above, $s_{n_{1}+1}$ is in $F$. Therefore by (3) we see that $F$ is in $\mathscr{I}_{n_{1}+1}$. Moreover, since $s_{n}$ is in $F$ for all $n \geqq n_{1}$, it follows from (3) that $F$ is in $\mathscr{I}_{n}$ for all $n>n_{1}$. Hence $\mathscr{F}_{n} \neq \varnothing$ for all $n>n_{1}$. This is a contradiction. Thus $\mathscr{F}_{n} \neq \varnothing$ for all $n \geqq n_{0}$. Therefore $\operatorname{In}(\tilde{r} \mid \pi) \subseteq \tilde{F}$. Conversely suppose that $\operatorname{In}(\tilde{r} \mid \pi) \cong \tilde{F}$. Then it follows from (3) and (4) that $\mathscr{I}_{n} \supseteqq \mathscr{F}_{n+1}$ and $\mathscr{F}_{n} \neq \varnothing$ for all $n \geqq n_{0}$. Since $\mathscr{F}_{n}$ is finite and $\mathscr{F}_{n} \neq \varnothing$, there exists $F$ in $\mathscr{F}$ such that $F$ is in $\mathscr{F}_{n}$ for all $n \geqq n_{0}$. Therefore $s_{n}$ is in $F$ for all $n \geqq n_{0}$. Thus $\operatorname{In}(r \mid \pi) \cong F$.

CASE 3. $i=4: \tilde{M}$ is defined as follows :

(1) $\tilde{S}=\{(s, \tilde{\mathscr{T}}) \mid \tilde{\mathscr{T}} \subseteq \mathscr{F}$ and for each $F$ in $\tilde{\mathscr{T}}, s$ is in $F\}$.

(2) $\tilde{s}_{0}=\left(s_{0}, \mathscr{I}_{0}\right)$, where $\mathscr{I}_{0}=\left\{F \mid F \in \mathscr{F}, s_{0} \in F\right\}$.

(3) If $\left(s_{1}, s_{2}\right)$ is in $\Delta(s, \sigma)$, then for all $\tilde{F} \subseteq \mathscr{F}, \Delta((s, \tilde{\mathscr{F}}), \sigma)$ contains $\left(\left(s_{1},\{F \mid F \in \tilde{T}\right.\right.$, $\left.\left.\left.s_{1} \in F\right\}\right),\left(s_{2},\left\{F \mid F \in \tilde{\mathscr{F}}, s_{2} \in F\right\}\right)\right)$.

(4) $\tilde{F}=\{(s, \tilde{\mathscr{I}}) \mid(s, \tilde{\mathscr{I}}) \in \tilde{S}$ with $\tilde{\mathscr{I}} \neq \varnothing\}$.

Let $t: T_{2} \rightarrow \Sigma$ be a $\Sigma$-tree. As in Case 2, by the one-to-one correspondence between the runs of $M$ on $t$ and the runs of $\tilde{M}$ on $t$, we denote by $r$ and $\tilde{r}$ the runs correspond- 
ing to each other. Let $\pi=\left\{x_{0}<x_{1}<\cdots x_{n}<\cdots\right\}$ be an arbitrary path in $T_{2}$ and let $\tilde{r}\left(x_{n}\right)=\left(s_{n}, \mathscr{I}_{n}\right)$ for $n \geqq 0$. Then we show the equivalence of the following two statements.

(5) There exists $F$ in $F$ such that $r(\pi) \leqq F$.

(6) $\tilde{r}(\pi) \subseteq \tilde{F}$.

Suppose that $r(\pi) \leqq F$ for some $F$ in $\mathscr{F}$. Since $r(\varepsilon)=s_{0}$ is in $F$, we see that $F$ is in $\mathscr{I}_{0}$. By induction we can see that $F$ is in $\mathscr{F}_{n}$ for all $n \geqq 0$. Hence $\mathscr{I}_{n} \neq \varnothing$ for all $n \geqq 0$. Therefore $\tilde{r}(\pi) \subseteq \tilde{F}$. Conversely, if $\tilde{r}(\pi) \subseteq \widetilde{F}$, then $\mathscr{I}_{n} \neq \varnothing$ for all $n \geqq 0$. Since $\mathscr{F}_{n} \supseteqq \mathscr{F}_{n+1}$ and $\mathscr{F}_{n} \neq \varnothing$ for $n \geqq 0$, there exists $F$ such that $F$ is in $\mathscr{F}_{n}$ for all $n \geqq 0$. Since $\tilde{r}\left(x_{n}\right)=$ $\left(r\left(x_{n}\right), \Phi_{n}\right)$, it follows from (3) that $r\left(x_{n}\right)$ is in $F$ for all $n \geqq 0$. Hence $r(\pi) \subseteq F$.

In Cases $1-3$, the construction of $\tilde{M}$ shows that if $M$ is deterministic then so is $\tilde{M}$.

By Theorem 3.1, we hereafter consider only finite tree automata with a single final set for $C_{i}$-acceptances for $i=1, \cdots, 4$.

For the acceptance conditions $C_{5}$ and $C_{6}$, the number of final sets affects the definability. We will discuss this matter in Section 6.

\section{Embedding Theorem}

Let $\Sigma=\{a, b\}$. We define $\iota: \Sigma^{T_{1}} \rightarrow \Sigma^{T_{2}}$ and $\rho: \Sigma^{T_{2}} \rightarrow \Sigma^{T_{1}}$ as follows: For $w$ in $\Sigma^{T_{1}}, \iota(w)$ is defined by $\iota(w)(x)=w(x)$ for $x$ in $T_{1}$ and $\iota(w)(x)=a$ for $x$ in $T_{2}-T_{1}$. For $t$ in $\Sigma^{r_{2}}$, we define $\rho(t)=t \mid T_{1}$. Namely, $\iota(w)$ is a $\Sigma$-tree whose leftmost path is the same as $w$ and other nodes are labelled with $a . \rho(t)$ is the $\omega$-word obtained by spelling out the labels of the leftmost path of $t$.

THEOREM 4.1. For $i=1, \cdots, 6$, if $L$ is in $\pi \mathscr{I}_{i}\left(\right.$ resp. $\left.\mathscr{D} \mathscr{I}_{i}\right)$, then $\rho(L)$ is in $\pi_{i}$ (resp. $\mathscr{D}_{i}$ ).

PROOF. CASE 1. $i=1$ : Given a finite tree automaton $M=\left\langle S, \Sigma, \Delta, s_{0}, F\right\rangle$ with $L=L_{1}(M)$, we construct a finite automaton $\tilde{M}=\left\langle\tilde{S}, \Sigma, \tilde{\Delta}, \tilde{s}_{0}, \tilde{F}\right\rangle$ as follows: For $s$ in $S$, let $M_{s}=\langle S, \Sigma, \Delta, s, F\rangle$ and let $S_{1}=\left\{s \in S \mid L_{1}\left(M_{s}\right) \neq \varnothing\right\}$. Then we define as follows :

(1) $\tilde{S}=S \cup\{\perp\}$, where $\perp \notin S$.

(2) $\tilde{S}_{0}=s_{0}$.

(3) For each $s$ in $S$ and $\sigma$ in $\Sigma, \tilde{\Delta}(s, \sigma)$ is equal to the set $\{p \mid$ There is $(p, q) \in$ $\Delta(s, \sigma)$ with $\left.q \in S_{1}\right\}$ if this set is not empty else $\{\perp\}$. And $\tilde{\Delta}(\perp, \sigma)=\{\perp\}$.

(4) $\tilde{F}=F$.

If $t$ is in $L$, then there exists a run $r$ of $M$ on $t$ such that for any path $\pi$ in $T_{2}$, $\operatorname{In}(r \mid \pi) \cap F \neq \varnothing$. Therefor $r\left(0^{n} 1\right)$ is in $S_{1}$ for all $n \geqq 0$. Hence $\tilde{r}$, defined by $\tilde{r}(x)=r(x)$ for $x$ in $T_{1}$, is a run of $\tilde{M}$ on $\rho(t)$. Moreover $\tilde{r}$ is a $C_{1}$-accepting run since $\operatorname{In}\left(r \mid \pi_{0}\right) \cap$ $F \neq \varnothing$ for the leftmost path $\pi_{0}$ in $T_{2}$. Thus $\rho(L) \leqq L_{1}(\tilde{M})$. Conversely if $w$ is in $L_{1}(\tilde{M})$, then it follows from the definitions of $S_{1}$ and $\tilde{\Delta}$ that there exist a $\Sigma$-tree $t$ and a $C_{1^{-}}$ accepting run of $M$ on $t$ such that $w=\rho(t)$. Therefore $L_{1}(\tilde{M}) \subseteq \rho(L)$. Thus $\rho(L)=L_{1}(\tilde{M})$.

CASE 2. $i=2$ : This case can be proved in the same way as Case 1.

CASE 3. $i=3$ : Let $M=\left\langle S, \Sigma, L, s_{0}, F\right\rangle$ be a finite tree automaton with $L=L_{3}(M)$. Without loss of generality, we may assume that $s_{0}$ is not in $F$. We construct a finite automaton $\tilde{M}=\left\langle\tilde{S}, \Sigma, \tilde{\Delta}, \tilde{S}_{0}, \tilde{F}\right\rangle$ satisfying $\rho(L)=L_{3}(\tilde{M})$ in the following way: Let $S_{3}=$ $\left\{s \in S \mid L_{3}\left(M_{s}\right) \neq \varnothing\right\}$. Notice that if $s$ is in $F$ then $L_{3}\left(M_{s}\right)=\Sigma^{T_{2}}$. Hence $F \cong S_{3}$. For each $s$ in $S$ and $\sigma$ in $\Sigma$, let $S_{(s, \sigma)}=\left\{p \mid\right.$ There is $(p, q) \in \Delta(s, \sigma)$ with $\left.q \in S_{3}\right\}$. 
(1) $\tilde{S}=S \cup\{\perp, T\}$, where $\perp, T \in S$.

(2) $\tilde{s}_{0}=s_{0}$.

(3) For each $s$ in $S$ and $\sigma$ in $\Sigma, \tilde{J}(s, \sigma)$ is defined by

$$
\tilde{J}(s, \sigma)= \begin{cases}\perp & \text { if } \quad S_{(s, \sigma)}=\varnothing \\ \top & \text { if } \quad S_{(s, \sigma)} \cap F \neq \varnothing \\ S_{(s, \sigma)} & \text { otherwise }\end{cases}
$$

and $\tilde{\mathcal{J}}(\perp, \sigma)=\{\perp\}, \tilde{J}\left(\top^{\top}, \sigma\right)=\{\top\}$.

(4) $\tilde{F}=\{T\}$.

Let $t$ be in $L$ and let $r$ be a $C_{3}$-accepting run of $M$ on $t$. Then we inductively define $\tilde{r}: T_{1} \rightarrow \tilde{S}$ as follows: Let $\tilde{r}(\varepsilon)=r(\varepsilon)=s_{0}$. Assume that $\tilde{r}\left(0^{n}\right)$ is defined. If $\tilde{r}\left(0^{n}\right)$ is in $S$, then two cases arise. First if $r\left(0^{n+1}\right)$ is in $F$, then $\tilde{r}\left(0^{n+1}\right)=T$. If $r\left(0^{n+1}\right)$ is $S-F$, then $\tilde{r}\left(0^{n+1}\right)=r\left(0^{n+1}\right)$. If $\tilde{r}\left(0^{n}\right)=\top$, then $\tilde{r}\left(0^{n+1}\right)=\top$. Since $r\left(\pi_{0}\right) \cap F \neq \varnothing$ for the leftmost path $\pi_{0}$, there exists an integer $n_{0} \geqq 0$ such that $\tilde{r}\left(0^{n}\right)$ is in $S-F$ for $0 \leqq n \leqq n_{0}$ and $\tilde{r}\left(0^{n}\right)$ $=T$ for all $n>n_{0}$. Since $r$ is a $C_{3}$-accepting run of $M$ on $t$ and since $r\left(0^{n}\right)$ is in $S-F$ for $0 \leqq n \leqq n_{0}, r\left(0^{n+1}\right) \in S_{(r(0 n), t(0 n))}$ for $0 \leqq n \leqq n_{0}$ and $r\left(0^{n_{0}+1}\right) \in S_{(r(0 m), t(0 m))} \cap F$, where $m=n_{0}$. Therefore $\tilde{r}$ is a $C_{3}$-accepting run of $\tilde{M}$ on $\rho(t)$. Hence $\rho(L) \leqq L_{3}(\tilde{M})$. Conversely, let $\tilde{r}$ be a $C_{3}$-accepting run of $\tilde{M}$ on $w$ in $L_{3}(\tilde{M})$. Then by (3) and (4), there exists an integer $n_{0} \geqq 0$ such that $\tilde{r}\left(0^{n}\right)$ is in $S-F$ for $0 \leqq n \leqq n_{0}$ and $\tilde{r}\left(0^{n}\right)=T$ for all $n>n_{0}$. Furthermore for each $n, 0 \leqq n \leqq n_{0}$, there is $q_{n}$ in $S_{3}$ such that $\left(\tilde{r}\left(0^{n+1}\right), q_{n}\right) \in \Delta\left(\tilde{r}\left(0^{n}\right), w\left(0^{n}\right)\right)$ and $\tilde{r}\left(0^{n_{0}+1}\right) \in F \subseteq S_{3}$. Therefore by the definition of $S_{3}$, we can see that there exist a $\Sigma$-tree $t$ with $\rho(t)=w$ and a $C_{3}$-accepting run of $M$ on $t$.

CASE 4. $i=4$ : This case can be proved in a manner similar to Case 1.

CASE 5. $i=5$ : For a finite tree automaton $M=\left\langle S, \Sigma, \Delta, s_{0}, \mathscr{I}\right\rangle$ with $L=L_{5}(M)$, we construct a finite automaton $\tilde{M}=\left\langle\widetilde{S}, \Sigma, \tilde{\Delta}, \tilde{s}_{0}, \tilde{\mathscr{F}}\right\rangle$ as follows : Let $S_{5}=\left\{s \in S \mid L_{5}\left(M_{s}\right) \neq \varnothing\right\}$. Then we define as follows:

(1) $\tilde{S}=S \cup\{\perp\}$, where $\perp \notin S$.

(2) $\tilde{s}_{0}=s_{0}$.

(3) For each $s$ in $S$ and $\sigma$ in $\Sigma, \tilde{J}(s, \sigma)$ is equal to the set $\{p \mid$ There is $(p, q) \in$ $\Delta(s, \sigma)$ with $\left.q \in S_{5}\right\}$ if this set is not empty else $\{\perp\}$. And $\tilde{\Delta}(\perp, \sigma)=\{\perp\}$.

(4) $\tilde{\mathscr{T}}=\mathscr{F}$.

Then by an argument similar to Case 1 , we can prove $\rho(L)=L_{5}(\tilde{M})$.

CASE 6. $i=6$ : Let $M=\left\langle S, \Sigma, \Delta, s_{0}, \mathscr{F}\right\rangle$ be a finite tree automaton satisfying $L=$ $L_{6}(M)$. For $\varnothing \neq Q \cong S$ and $s$ in $S$, let $\mathscr{T}_{Q}=\{F \subseteq S \mid F \cup Q \subseteq \mathscr{F}\}$ and let $M_{(Q, s)}=\langle S, \Sigma, \Delta$, $\left.s, \mathscr{I}_{Q}\right\rangle$. Then let $S_{Q}=\left\{s \in Q \mid L_{6}\left(M_{(Q, s)}\right) \neq \varnothing\right\}$. Without loss of generality, we may assume that every $F$ in $\mathscr{F}$ contains the initial state $s_{0}$. For $F$ in $\mathscr{I}$, an $F$-chain is a set $\left\{S_{i} \mid 0 \leqq i \leqq n\right\}$ of subsets of $F$ such that $S_{0}=\left\{s_{0}\right\}, S_{n}=F, S_{i} \varsubsetneqq S_{i+1}$ and $\left|S_{i}\right|=i+1$ for $0 \leqq i \leqq n$. Then let $\mathcal{S}_{F}$ be the set of all $F$-chains. We define a finite automaton $\tilde{M}=$ $\left\langle\widetilde{S}, \Sigma, \tilde{\Delta}, \tilde{s}_{0}, \tilde{\mathcal{I}}\right\rangle$ as follows :

(1) $\tilde{S}=S \times P(S) \cup\{\perp\}$.

(2) $\tilde{s}_{0}=\left(s_{0},\left\{s_{0}\right\}\right)$.

(3) For each $s$ in $S, \sigma$ in $\Sigma$ and $Q \subseteq S, \tilde{J}((s, Q), \sigma)$ is equal to the set $\{(p, Q \cup\{p\}) \mid$ There is $(p, q) \in \Delta(s, \sigma)$ with $\left.q \in S_{Q \cup\{q\}}\right\}$ if this set is not empty else $\{\perp\}$. And 
$\tilde{\Delta}(\perp, \sigma)=\{\perp\}$.

(4) $\tilde{\mathscr{T}}=\left\{G \leqq S \times P(S) \mid p_{1}(G)=F\right.$ and $p_{2}(G) \in S_{F}$ for some $F$ in $\left.\mathscr{I}\right\}$, where $p_{1}$ and $p_{2}$ are the projections to the first and second components, respectively.

For $t$ in $L_{6}(M)$, let $r$ be a $C_{6}$-accepting run of $M$ on $t$. Let $s_{n}=r\left(0^{n}\right)$ and $F_{n}=\left\{s_{i} \mid 0 \leqq\right.$ $i \leqq n\}$ for $n \geqq 0$. Then we define $\tilde{r}: T_{1} \rightarrow \tilde{S}$ by $\tilde{r}\left(0^{n}\right)=\left(s_{n}, F_{n}\right)$ for $n \geqq 0$. We show that $\tilde{r}$ is a $C_{6}$-accepting run of $\tilde{M}$ on $\rho(t)$. First note that $\tilde{r}(\varepsilon)=\left(s_{0},\left\{s_{0}\right\}\right)$. For each $n \geqq 0$, consider the transition $\left(r\left(0^{n+1}\right), r\left(0^{n} 1\right)\right) \in \Delta\left(r\left(0^{n}\right), t\left(0^{n}\right)\right)$. Since $r$ is a $C_{6}$-accepting run of $M$ on $t, r\left(0^{n} 1\right)$ is in $S_{F_{n}} \cup_{\left(r\left(0^{n} 1\right) !\right.}$. Therefore $\tilde{r}$ is a run of $\tilde{M}$ on $\rho(t)$. Since $r\left(\pi_{0}\right)$ is in $\mathscr{I}$ for the leftmost path $\pi_{0}$ in $T_{2}, F=\left\{s_{n} \mid n \geqq 0\right\}$ is in $\mathscr{F}$. Since $F_{0}=\left\{s_{0}\right\}, F_{n} \leqq F_{n+1}$ for all $n \geqq 0$ and $F=\bigcup_{n=0}^{\infty} F_{n}$, the set $\left\{\left(s_{n}, F_{n}\right) \mid n \geqq 0\right\}$ is in $\tilde{\mathcal{F}}$. Hence $\tilde{r}$ is a $C_{6}$-accepting run of $\tilde{M}$ on $\rho(t)$. Thus $\rho(L) \leqq L_{6}(\tilde{M})$. Conversely, for a $C_{6}$-accepting run $\tilde{r}$ of $\tilde{M}$ on $w$ in $L_{6}(\tilde{M})$, we can find a $\Sigma$-tree $t$ with $\rho(t)=w$ and a $C_{6}$-accepting run of $M$ on $t$ by using the definitions of $S_{Q}$ and $\tilde{J}$. Thus $L_{6}(\tilde{M}) \leqq \rho(L)$.

In Cases $1-6$, it is obvious that if $M$ is deterministic then so is $\tilde{M}$.

REMARK. For a finite tree automaton $M=\left\langle S, \Sigma, \Delta, s_{0}, \mathscr{T}\right\rangle$, we define a finite automaton $M^{\prime}=\left\langle S, \Sigma, \Delta^{\prime}, s_{0}, \mathscr{F}\right\rangle$ by setting $\Delta^{\prime}(s, \sigma)=\{p \mid(p, q) \in \Delta(s, \sigma)\}$ for $s$ in $S$ and $\sigma$ in $\Sigma$. Then $M^{\prime}$ does not necessarily accept $\rho\left(L_{i}(M)\right)$ in the sense of $C_{i}$ for $i=1, \cdots, 6$. Namely, the definitions of $\tilde{M}$ in the proof of Theorem 4.1 are essential. For example, let $M_{i}=\left\langle S, \Sigma, \Delta_{i}, s_{0}, F_{i}\right\rangle$ be

$$
\begin{aligned}
& S=\left\{s_{0}, s_{1}\right\}, \\
& \Sigma=\{a\}, \\
& \Delta_{i}\left(s_{0}, a\right)= \begin{cases}\left(s_{1}, s_{0}\right) & i=3 \\
\left(s_{0}, s_{1}\right) & i \neq 3 .\end{cases} \\
& \Delta_{i}\left(s_{1}, a\right)=\left(s_{1}, s_{1}\right) \\
& \text { for } i=1, \cdots, 6 . \\
& F_{i}= \begin{cases}\left\{s_{1}\right\} & i=3 \\
\left\{s_{0}\right\} & i \neq 3 .\end{cases}
\end{aligned}
$$

Then $L_{i}\left(M_{i}\right)=\varnothing$ for each $i=1, \cdots, 6$. However, $L_{i}\left(M_{i}^{\prime}\right) \neq \varnothing$ for each $i=1, \cdots, 6$.

REMARK. In the proof of Theorem $4.1,|\tilde{F}|=|\mathscr{F}|$ for $i=1, \cdots, 5$. However, for the $C_{6}$-acceptance, the size of $\tilde{\Phi}$ is increased in the construction of $\tilde{M}$.

The following embedding theorem establishes the relationship between $\mathscr{N}_{i}$ (resp. $\mathscr{D}_{i}$ ) and $\mathscr{N} \mathscr{I}_{i}\left(\right.$ resp. $\left.\mathscr{D} \mathscr{I}_{i}\right)$ for $i=1, \cdots, 6$.

THEOREM 4.2. For $i=1, \cdots, 6$ and $L \cong \Sigma^{T_{1}}, L$ is in $\eta_{i}\left(\right.$ resp. $\left.\mathscr{D}_{i}\right)$ if and only if $\iota(L)$ is in $\mathscr{N I}_{i}\left(\right.$ resp. $\left.\mathscr{D} \mathscr{I}_{i}\right)$.

PROOF. For $L \subseteq \Sigma^{T_{1}}$, let $M=\left\langle S, \Sigma, \Delta, s_{0}, \mathscr{I}\right\rangle$ be a finite automaton such that $L=$ $L_{i}(M)$. Then a finite tree automaton $\tilde{M}=\left\langle\tilde{S}, \Sigma, \tilde{\Delta}, \tilde{S}_{0}, \tilde{\mathscr{F}}\right\rangle$ which accepts $\iota(L)$ in the sense of $C_{i}$ is constructed as follows:

(1) $\tilde{S}=S \cup\{\perp, T\}$, where $\perp, T \in S$.

(2) $\tilde{S}_{0}=s_{0}$.

(3) For each $s$ in $S$, 


$$
\begin{gathered}
\tilde{J}(s, \sigma)=\{(p, T) \mid p \in \Delta(s, \sigma)\} \quad \text { for } \sigma \text { in } \Sigma, \\
\tilde{J}(T, a)=\{(T, T)\}, \Delta(T, b)=\{(\perp, \perp)\}, \\
\tilde{J}(\perp, \sigma)=\{(\perp, \perp)\} \quad \text { for } \sigma \text { in } \Sigma . \\
\text { (4) } \tilde{\mathscr{I}}= \begin{cases}\mathscr{I} \cup\{\{T\}\} & \text { for } i=1,2,3,5 \\
\mathscr{I} \cup\left\{\left\{s_{0}, T\right\}\right\} & \text { for } i=4,6 .\end{cases}
\end{gathered}
$$

Notice that if $M$ is deterministic then so is $\tilde{M}$.

The converse direction follows from Theorem 4.1.

\section{Comparison of the Classes Concerned}

This section relates the classes $\Re I_{i}$ and $\mathscr{D} \mathscr{I}_{i}$ for $i=1, \cdots, 6$. The results are summarized in the lattice diagram in Fig. 1, where an unbroken line indicates a proper containment and a broken line means a containment whose properness is unknown. One can also draw a similar diagram for the deterministic classes.

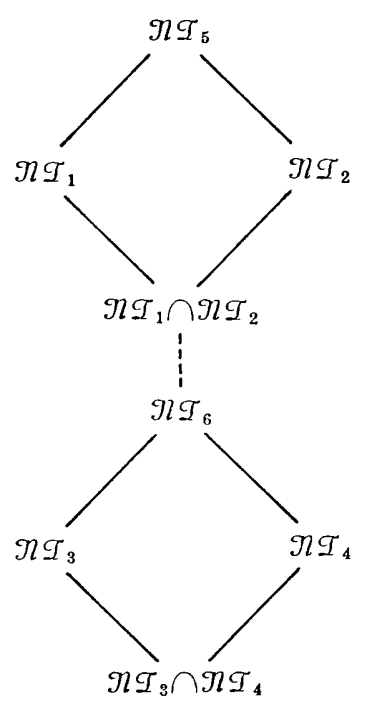

Fig. 1.

Notation. Let $t$ be in $\Sigma^{T_{2}}$. For a path $\pi=\left\{x_{0}<x_{1}<\cdots x_{n}<\cdots\right\}$ in $T_{2}$, we denote by $[t \mid \pi] \in \Sigma^{T_{1}}$ a function defined by $[t \mid \pi]\left(0^{n}\right)=t\left(x_{n}\right)$ for $n \geqq 0$.

PROPOSITION 5.1. (1) $\Re_{I_{1}}$ and $\Re I_{2}$ are incomparable.

(2) $\mathscr{D} \mathscr{I}_{1}$ and $\mathscr{D} \mathscr{I}_{2}$ are incomparable.

Proof. Let $\Sigma=\{a, b\}$ and let

$$
\begin{aligned}
& L_{1}=\iota\left(L^{\prime}\right), \text { where } L^{\prime}=\left(b a^{*}\right)^{\omega} \subseteq \Sigma^{T_{1}}, \\
& L_{2}=\left\{t \in \Sigma^{T_{2}} \mid[t \mid \pi] \in \Sigma^{*} a^{\omega} \subseteq \Sigma^{T_{1}} \text { for every path } \pi \text { in } T_{2}\right\} .
\end{aligned}
$$

We can easily construct a deterministic finite tree automaton which accepts $L_{i}$ in the 
sense of $C_{i}$ for $i=1,2$. Since $L^{\prime}$ is known not to be in $F_{\sigma}^{R}[2,11]$ and since $\Re_{2}=F_{\sigma}^{R}$ from Table 1, it follows from Theorem 4.2 that $L_{1}$ is not in $\Re I_{2}$. On the other hand, Rabin [7] has proved that $L_{2}$ is not in $\Re I_{1}$. Hence $L_{1}$ is in $\mathscr{D} \mathscr{I}_{1}-\Re I_{2}$ and $L_{2}$ is in $\mathscr{D} \mathscr{I}_{2}-\eta I_{1}$.

PROPOSITION 5.2. (1) $\mathfrak{n I}_{3}$ and $\mathfrak{n I}_{4}$ are incomparable.

(2) $\mathscr{D} I_{3}$ and $\mathscr{D I} \mathscr{I}_{4}$ are incomparable.

Proof. These results follow from Theorem 4.2 and the fact that $G^{R}$ and $F^{R}$ in Table 1 are incomparable.

THEOREM 5.3. (1) $\Re \mathscr{I}_{3} \cup \Re I_{4} \subsetneq \Re I_{6}$.

(2) $\mathscr{D I}_{3} \cup \mathscr{D} \mathscr{I}_{4} \subsetneq \mathscr{D} \mathscr{I}_{6}$.

Proof. By Theorem 3.1, we deal with a finite tree automaton $M=\left\langle S, \Sigma, \Delta, s_{0}, F\right\rangle$ with just one final set. Then we consider finite tree automata $M_{0}=\left\langle S, \Sigma, \Delta, s_{0}, \mathscr{T}_{0}\right\rangle$ and $M_{1}=\left\langle S, \Sigma, \Delta, s_{0}, \mathscr{I}_{1}\right\rangle$, where $\mathscr{I}_{0}=\{G \mid G \cap F \neq \varnothing, G \subseteq S\}$ and $\mathscr{F}_{1}=\{G \mid \varnothing \neq G \subseteq F\}$. Then it is not difficult to see that $L_{3}(M)=L_{6}\left(M_{0}\right)$ and $L_{4}(M)=L_{6}\left(M_{1}\right)$. By the definition, it is obvious that if $M$ is deterministic then so are $M_{0}$ and $M_{1}$. The properness of the inclusions follows from Theorem 4.2 and the fact that $G^{R} \cup F^{R} \subsetneq G_{\delta}^{R} \cap F_{\sigma}^{R}$ in Table 1.

THEOREM 5.4 [5]. (1) $\mathscr{n} \mathscr{I}_{6} \leqq \Re I_{1} \cap \mathscr{I I}_{2}$.

(2) $\mathscr{D} I_{6} \cong \mathscr{D} I_{1} \cap \mathscr{D} I_{2}$.

Proof. Let $M=\left\langle S, \Sigma, \Delta, s_{0}, \mathscr{I}\right\rangle$ be a finite tree automaton. We construct a finite tree automaton $M^{\prime}=\left\langle S^{\prime}, \Sigma, \Delta^{\prime}, s_{0}^{\prime}, F^{\prime}\right\rangle$ such that $T_{6}(M)=T_{1}\left(M^{\prime}\right)=T_{2}\left(M^{\prime}\right)$ as follows :

(1) $S^{\prime}=S \times P(S)$.

(2) $s_{0}^{\prime}=\left(s_{0},\left\{s_{0}\right\}\right)$.

(3) For each $s \in S, Q \subseteq S$ and $\sigma \in \Sigma, \Delta^{\prime}((s, Q), \sigma)=\left\{\left(\left(p, Q_{1}\right),\left(q, Q_{2}\right)\right) \mid(p, q) \in \Delta(s, \sigma)\right.$, $\left.Q_{1}=Q \cup\{p\}, Q_{2}=Q \cup\{q\}\right\}$.

(4) $F^{\prime}=\{(s, F) \mid s \in S, F \in \mathscr{I}\}$.

As simulating $M, M^{\prime}$ stores the states occurring on the path from the root to the current node in the second component of its state. Let $t$ be in $L_{1}\left(M^{\prime}\right)$ and let $r^{\prime}$ be a $C_{1}$-accepting run of $M^{\prime}$ on $t$. Then we define $r$ by $r(x)=p_{1}\left(r^{\prime}(x)\right)$, where $p_{1}$ is the projection to the first component. By (3), $r$ is a run of $M$ on $t$. We show that $r$ is a $C_{6}$-accepting run of $M$ on $t$. For an arbitrary path $\pi=\left\{x_{0}<x_{1}<\cdots x_{n}<\cdots\right\}$ in $T_{2}$, let $r^{\prime}\left(x_{n}\right)=\left(s_{n}, F_{n}\right)$ for $n \geqq 0$. Then by definition, we see that $F_{n} \leqq F_{n+1}$ for $n \geqq 0$. Therefore there exists an integer $n_{0}$ such that $F_{n_{0}}=F_{n}$ for all $n \geqq n_{0}$. Note that $F_{n_{0}}=$ $\left\{s_{n} \mid n \geqq 0\right\}$. Since $r^{\prime}$ is a $C_{1}$-accepting run, $\operatorname{In}\left(r^{\prime} \mid \pi\right) \cap F^{\prime} \neq \varnothing$. Therefore there is $(s, F)$ in $F^{\prime}$ such that $(s, F)=\left(s_{n}, F_{n}\right)$ for infinitely many $n \geqq 0$. Since $F_{n_{0}}=F_{n}$ for all $n \geqq n_{0}$, $F=F_{n_{0}}$. Since $F_{n_{0}}=r(\pi), r(\pi)$ is in $\mathscr{F}$. Hence $r$ is a $C_{6}$-accepting run of $M$ on $t$. Thus $L_{1}\left(M^{\prime}\right) \leqq L_{6}(M)$.

Conversely, let $t$ be in $L_{6}(M)$ and let $r$ be a $C_{6}$-accepting run of $M$ on $t$. We define $r^{\prime}$ inductively. Let $r^{\prime}(\varepsilon)=\left(s_{0},\left\{s_{0}\right\}\right)$. If $r^{\prime}(x)=(s, Q)$ is defined, then let $r^{\prime}(x 0)=$ $(r(x 0), Q \cup\{r(x 0)\})$ and $r^{\prime}(x 1)=(r(x 1), Q \cup\{r(x 1)\})$. Then $r^{\prime}$ is a run of $M^{\prime}$ on $t$ since $r$ is a run of $M$ on $t$. Let $\pi=\left\{x_{0}<x_{1}<\cdots x_{n}<\cdots\right\}$ be an arbitrary path in $T_{2}$. Let $r^{\prime}\left(x_{n}\right)=\left(s_{n}, F_{n}\right)$ for $n \geqq 0$. Since $r$ is a $C_{6}$-accepting run, $r(\pi)=F$ for some $F$ in $\mathscr{T}$. Since $F_{n} \leqq F_{n+1}$ and $F_{n}=\left\{s_{i} \mid 0 \leqq i \leqq n\right\}$, there exists an integer $n_{0}$ such that $F=F_{n}$ for all $n \geqq n_{0}$. Since $\left\{s_{n} \mid n \geqq n_{0}\right\}$ is finite, there exists $s$ in $S$ such that $(s, F)=\left(s_{n}, F_{n}\right)$ for 
infinitely many $n \geqq 0$. Therefore $\operatorname{In}\left(r^{\prime} \mid \pi\right) \cap F^{\prime} \neq \varnothing$. Thus $r^{\prime}$ is a $C_{1}$-accepting run of $M^{\prime}$ on $t$. Hence $L_{6}(M) \subseteq L_{1}\left(M^{\prime}\right)$. Thus $L_{6}(M)=L_{1}\left(M^{\prime}\right)$.

We now prove $L_{2}\left(M^{\prime}\right)=L_{6}(M)$. We use the same definitions as those in the proof of $L_{1}\left(M^{\prime}\right)=L_{6}(M)$. Suppose that $r^{\prime}$ be a $C_{2}$-accepting run of $M^{\prime}$ on $t$. Then for every path $\pi, \operatorname{In}\left(r^{\prime} \mid \pi\right) \subseteq F^{\prime}$. Since $\operatorname{In}\left(r^{\prime} \mid \pi\right) \neq \varnothing$, there exists $(s, F)$ in $\mathscr{I}^{\prime}$ such that $(s, F)=$ $\left(s_{n}, F_{n}\right)$ for infinitely many $n \geqq 0$. Since $F_{n} \subseteq F_{n+1}$ for $n \geqq 0$, there exists an integer $n_{0}$ such that $F=F_{n}$ for all $n \geqq n_{0}$. Therefore the run $r$ defined by $r(x)=p_{1}\left(r^{\prime}(x)\right)$ for $x$ in $T_{2}$ has the property that $r(\pi)=F$. Thus $r$ is a $C_{6}$-accepting run of $M$ on $t$. Hence $L_{2}\left(M^{\prime}\right) \subseteq L_{6}(M)$. To see the converse inclusion, it may be sufficient to note that in the proof of $L_{6}(M) \subseteq L_{1}\left(M^{\prime}\right)$ we have $\left(s_{n}, F_{n}\right)=\left(s_{n}, F\right)$ for all $n \geqq n_{0}$, where $F$ is in $\mathscr{F}$. Thus $\operatorname{In}\left(r^{\prime} \mid \pi\right) \leqq F^{\prime}$. Therefore $r^{\prime}$ is also a $C_{2}$-accepting run of $M^{\prime}$ on $t$. Hence $L_{6}(M)$ $\subseteq L_{2}\left(M^{\prime}\right)$.

By the construction, it is also obvious that if $M$ is deterministic then so is $M^{\prime}$.

REMARK. It is an open problem whether the inclusion is proper in Theorem 5.4.

THEOREM 5.5. (1) $\mathfrak{M I}_{1} \cup \mathfrak{N I}_{2} \subsetneq \Re \mathscr{I}_{5}$

(2) $\mathscr{D} I_{1} \cup \mathscr{D} \mathscr{I}_{2} \subsetneq \mathscr{D I} \mathscr{I}_{5}$.

Proof. Given a finite tree automaton $M=\left\langle S, \Sigma, \Delta, s_{0}, F\right\rangle$, we define in the same way as Theorem 5.3 finite tree automata $M_{0}=\left\langle S, \Sigma, \Delta, s_{0}, \mathscr{F}_{0}\right\rangle$ and $M_{1}=\left\langle S, \Sigma, \Delta, s_{0}, \mathscr{F}_{1}\right\rangle$, where $\mathscr{F}_{0}=\{G \mid G \cap F \neq \varnothing, G \leqq S\}$ and $\mathscr{T}_{1}=\{G \mid \varnothing \neq G \leqq F\}$. Then we can see that $L_{1}(M)$ $=L_{5}\left(M_{0}\right)$ and $L_{2}(M)=L_{5}\left(M_{1}\right)$.

We now prove the properness of the inclusions. Let $\Sigma=\{a, b\}$ and let

$$
\begin{aligned}
& L_{1}=\iota\left(L^{\prime}\right), \text { where } L^{\prime}=\left(b a^{*}\right)^{\omega} \cong \Sigma^{T_{1}}, \\
& L_{2}=\left\{t \in \Sigma^{T_{2}} \mid[t \mid \pi] \in a \Sigma^{*} a^{\omega} \text { for every path } \pi \cong T_{2}\right\} .
\end{aligned}
$$

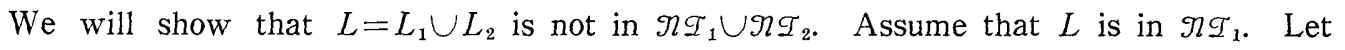
$M=\left\langle S, \Sigma, \Delta, s_{0}, F\right\rangle$ be a finite tree automaton accepting $L$ in the sense of $C_{1}$. One can modify $M$ to get a finite tree automaton $M_{2}$ which accepts $L_{2}$ in the sense of $C_{1}$ since for each $t \in L_{1}, t(\varepsilon)=b$ and on the other hand for each $t \in L_{2}, t(\varepsilon)=a$. By the result due to Rabin [7], $L_{2}$ is not in $\Re_{I_{1}}$. Hence $L$ is not in $\Re I_{1}$. Now assume that $L$ is in $\mathscr{N I}_{2}$. Then by the same consideration, one can get a finite tree automaton which accepts $L_{1}$ in the sense of $C_{2}$. In the same way as Proposition 5.1, this yields a contradiction. Hence $L$ is not in $\Re I_{2}$. Therefore $L$ in not in $\mathscr{N} \mathscr{I}_{1} \cup \mathfrak{M I}_{2}$.

We can easily construct a deterministic finite tree automaton which accepts $L$ in the sense of $C_{5}$.

\section{Structural Measures for Finite Tree Automata}

In this section we introduce two structural measures for finite tree automata to give further observations on $\Re \mathscr{I}_{i}$ for $i=1, \cdots, 6$. Let $M=\left\langle S, \Sigma, \Delta, s_{0}, \mathscr{F}\right\rangle$ be a finite tree automaton. The first measure is the nondeterministic degree of $M$, denoted by ndeg $(M)$, which is defined to be $\max \{|\Delta(s, \sigma)| \mid s \in S, \sigma \in \Sigma\}$. The second measure is the number $|\mathscr{F}|$ of final sets. As shown in Section 3, the size of $\mathscr{T}$ does not affect the $C_{i}$-acceptances for $i=1, \cdots, 4$.

Definition. For $i=1, \cdots, 6$ and an integer $k \geqq 1$, a set $L$ in $\mathscr{M} \mathscr{I}_{i}$ is said to be of 
nondeterministic degree $k$ if $k=\min \left\{\right.$ ndeg $\left.(M) \mid L=L_{i}(M)\right\}$. Then we define

$$
k-\mathscr{N} I_{i}=\left\{L \in \Re I_{i} \mid L \text { is of nondeterministic degree } l \text { with } 1 \leqq l \leqq k\right\} .
$$

A deterministic finite automaton is of nondeterministic degree one. The following theorem refines the result in [9] which separates the nondeterminsitic classes from the deterministic classes.

THEOREM 6.1. For $i=1, \cdots, 6$ and for $k \geqq 1, k-\Re I_{i} \varsubsetneqq(k+1)-\Re I_{i}$.

Proof. Let $\Sigma=\{a, b\}$. For $n \geqq 1$, let $t_{n}$ be the $\Sigma$-tree defined by

$$
t_{n}(x)= \begin{cases}b & \text { if }|x|=n \\ a & \text { otherwise }\end{cases}
$$

Let $L_{k}=\left\{t_{1}, \cdots, t_{k}\right\}$. We show that $L_{k+1}$ is in $(k+1)-\eta I_{i}$ but not in $k-\eta I_{i}$ for $i=1, \cdots, 6$. One can easily construct a nondeterministic finite tree automaton of nondeterministic degree $k+1$ which accepts $L_{k+1}$ in the sense of $C_{i}$. Suppose that $L_{k+1}$ is accepted in the sence of $C_{i}$ by a nondeterministic finite tree automaton $M=\langle S, \Sigma, \Delta$, $s_{0}, \mathscr{F}>$ with $n \operatorname{deg}(M) \leqq k$. Let $r_{n}$ be a $C_{i}$-accepting run of $M$ on $t_{n}$ for $n=1, \cdots, k+1$. Since $\left|\Delta\left(s_{0}, a\right)\right| \leqq k$, there exist $m, n, 1 \leqq m<n \leqq k+1$, such that $r_{m}(\varepsilon)=r_{n}(\varepsilon)=s_{0}$ and $\left(r_{m}(0), r_{m}(1)\right)=\left(r_{n}(0), r_{n}(1)\right)$. Let $t$ be a $\Sigma$-tree defined by

$$
t(x)= \begin{cases}b & \text { if either }|x|=m \text { and } 0 \leqq x \text { or }|x|=n \text { and } 1 \leqq x \\ a & \text { otherwise }\end{cases}
$$

Then from $r_{m}$ and $r_{n}$ we can construct a $C_{i}$-accepting run of $M$ on $t$ in the following way. We prune the subtree at the node 1 from $r_{m}$ and instead of it we graft the subtree of $r_{n}$ at the node 1 . Then the resulting run is also a $C_{i}$-accepting run of $M$ on $t$. However, $t$ does not belong to $L_{k+1}$, a contradiction. Hence $L_{k+1}$ is not in $k-\Re I_{i}$.

Definition. The Muller degree of a language $L$ in $\Re I_{5}$, denoted by $m \operatorname{deg}(L)$, is the minimum number of final sets sufficient for its acceptance in the sense of $C_{5}$, i.e.

$$
m \operatorname{deg}(L)=\min \left\{k \mid L=L_{5}(M) \text { for } M=\left\langle S, \Sigma, \Delta, s_{0}, \mathscr{F}\right\rangle \text { with } k=|\mathscr{I}|\right\} .
$$

THEOREM 6.2. For each $k \geqq 0$, there exists a language $L_{k}$ in $\Re I_{5}$ such that $k=$ $\operatorname{mdeg}\left(L_{k}\right)$.

PROOF. For $n \geqq 1$, let $t_{n}$ be the $\sum$-tree such that $\left[t_{n} \mid \pi\right]=\left(a^{n} b\right)^{\omega}$ for each path $\pi$ in $T_{2}$. Let $L_{0}=\varnothing$ and $L_{k}=\left\{t_{1}, \cdots, t_{k}\right\}$ for $k \geqq 1$. It is clear that $m \operatorname{deg}\left(L_{0}\right)=0$. We will prove that $m \operatorname{deg}\left(L_{k}\right)=k$ for $k \geqq 1$. One can easily construct a deterministic finite tree automaton $M=\left\langle S, \Sigma, \Delta, s_{0}, \mathscr{F}\right\rangle$ such that $k=|\mathscr{F}|$ and $L_{k}=L_{5}(M)$. Thus $\operatorname{mdeg}\left(L_{k}\right) \leqq k$. Assume that $m \operatorname{deg}\left(L_{k}\right)=m<k$. Then there exists a nondeterministic finite tree automaton $M=\left\langle S, \Sigma, \Delta, s_{0}, \mathscr{T}\right\rangle$ such that $m=|\mathscr{F}|$ and $L_{k}=L_{5}(M)$. Then by Theorem 4.1, there exists a finite automaton $\tilde{M}=\left\langle\tilde{S}, \Sigma, \tilde{\Delta}, \tilde{s}_{0}, \tilde{\mathscr{I}}\right\rangle$ such that $m=|\tilde{\mathscr{F}}|$ and $\rho\left(L_{k}\right)=L_{5}(\tilde{M})$ because the construction of $\tilde{M}$ from $M$ preserves the number of final sets. Since $\rho\left(L_{k}\right)$ $=(a b)^{\omega} \cup\left(a^{2} b\right)^{\omega} \cup \cdots \cup\left(a^{k} b\right)^{\omega}$ and $m<k$, there exist $i \neq j$ such that both $\left(a^{i} b\right)^{\omega}$ and $\left(a^{j} b\right)^{\omega}$ can be accepted with the same final set $F$ in $\tilde{\mathscr{I}}$. Then there is $w \in \Sigma^{*}$ such that $a^{i} b w\left(a^{j} b\right)^{\omega}$ can be accepted by $\tilde{M}$ with $F$ in the sense of $C_{5}$. This is a contradiction. Hence $\operatorname{mdeg}\left(L_{k}\right)=k$. 
REMARK. Theorem 6.2 still holds when we restrict the case to the deterministic $C_{5}$-acceptance. By the results in Section 3, we can also define a similar degree for a language in $\Re I_{6}$. Although the language $L_{k}$ defined in the proof of Theorem 6.2 also seems to be a candidate which has exactly degree $k$ in this case, we have not proved this yet.

\section{Concluding Remarks}

By the results in Sections 4-6, the class $\Re_{\mathcal{I}_{5}}$ that is studied in [6] is the largest one among the classes defined by finite tree automata. This class is closed under Boolean operations [6] and for each $L$ in $\Re_{5}, \rho(L)$ is an $\omega$-regular language. Hence the class $\Re I_{5}$ may be considered to be a natural extension of the class of $\omega$-regular languages to infinite tree languages.

In [5], Moriya has characterized $\mathscr{N} \mathscr{I}_{3}$ and $\mathscr{N} \mathscr{I}_{4}$ in terms of general topology on $\Sigma^{T_{2}}$. We have not considered this approach in detail.

We have ignored the classes $\mathscr{N}_{i}^{(k)}$ for $k>2$. However, the arguments in this paper are easily applicable to $\Re_{i}^{(k)}$ and we can get similar results for $\eta_{i}^{(k)}$ for $k>2$. For instance, Theorems 4.1 and 4.2 can be generalized as follows: Let $\iota_{k}: \Sigma^{T_{k}} \rightarrow \Sigma^{T_{k+1}}$ be a function defined by $\iota_{k}(t)(x)=t(x)$ if $x$ is in $T_{k}$ and $\iota_{k}(t)(x)=a$ if $x$ is in $T_{k+1}-T_{k}$. Let $\rho_{k}: \Sigma^{T_{k+1}} \rightarrow \Sigma^{T_{k}}$ be a function defined by $\rho_{k}(t)=t \mid T_{k}$ for $t$ in $T_{k+1}$. Then we have the following generalibations:

THEOREM 4.1'. If $L$ is in $\Re_{i}^{(k+1)}$, then $\rho_{k}(L)$ is in $\Re_{i}^{(k)}$ for $i=1, \cdots, 6$.

THEOREM 4.2'. $L$ is in $\Re_{i}^{(k)}$ if ond only if $\iota_{k}(L)$ is in $\Re_{i}^{(k+1)}$ for $i=1, \cdots, 6$.

\section{References}

[1] BüchI, J.R.: On a decision method in restricted second order arithmetic. Proc. Methodology and Philosophy of Science, Stanford, Calif., (1960), 1-11.

[2] Landweber, L.H.: Decision problems for w-automata. Math. Systems Theory 4 (1969), 376-384.

[3] McNaughton, R.: Testing and generating infinite sequences by a finite automaton. Inform. Contr. 9 (1966), 521-530.

[4] MiYano, S. and HaYashi, T.: Alternating finite automata on $\omega$-words. to appear in Theoret. Comput. Sci.

[5] MORIYA, T.: Some classes of infinite tree languages (I). manuscript, LA symposium, (1984).

[6] Rabin, M.O.: Decidability of second-order theories and automata on infinite trees. Trans. Amer. Math. Soc. 141 (1969), 1-35.

[7] Rabin, M.O.: Weakly definable relations and special automata. Math. Logic and Foundations of Set Theory, Y. Bar-Hillel, North-Holland, Amsterdam, (1970), 1-23.

[8] Rabin, M.O.: Automata on infinite objects and Church's problem. AMS Regional Conference Series in Mathematics, No. 13 (1972), 1-22.

[9] SAOUd, A.: Infinitary tree languages recognized by w-automata. Inform. Process. Lett. 18 (1984), 15-19.

[10] StAiger, L. and WAgner, K.: Automatentheoretische und automatenfreie Charakterisierungen topologischer Klassen regulärer Folgenmengen. EIK 10 (1974), 379-392.

[11] TAKahashi, M. and Yamasaki, H.: A note on w-regular languages. Theoret. Comput. Sci. 23 (1983), 217-225.

[12] WAG.Ner, K.: On w-regular sets. Inform. Contr. 43 (1979), 123-177.

Communicated by S. Arikawa

Received September 6, 1984 